\title{
Preservation of spatial memory and neuroprotection by the fatty acid amide hydrolase inhibitor URB597 in a rat model of vascular dementia
}

\author{
Da-Peng Wang ${ }^{1,2}$, Qi Lin ${ }^{3}$, Kai Kang ${ }^{4}$, Yi-Fang Wu ${ }^{1}$, Shao-Hua Su ${ }^{1}$, Jian Hai ${ }^{1}$ \\ ${ }^{1}$ Department of Neurosurgery, Tong Ji Hospital, Tong Ji University School of Medicine, Shanghai, China; ${ }^{2}$ Department of Neurology and \\ Neurosurgery, Montreal Neurological Institute and Hospital, McGill University, Montreal, Quebec, Canada; ${ }^{3}$ Department of Pharmacy, Institutes \\ of Medical Sciences, Shanghai Jiao Tong University School of Medicine, Shanghai, China; ${ }^{4}$ Department of Research and Surveillance Evaluation, \\ Shanghai Center for Health Promotion, Shanghai, China \\ Contributions: (I) Conception and design: DP Wang, J Hai; (II) Administrative support: J Hai; (III) Provision of study materials or patients: J Hai; \\ (IV) Collection and assembly of data: Q Lin, YF Wu, SH Su; (V) Data analysis and interpretation: DP Wang, K Kang; (VI) Manuscript writing: All \\ authors; (VII) Final approval of manuscript: All authors. \\ Correspondence to: Da-Peng Wang; Jian Hai. No. 389, Xincun Road, Putuo District, Shanghai 200065, China. \\ Email:wdpboj@126.com; haijiandoct@zoho.com.cn.
}

Background: Chronic cerebral hypoperfusion $(\mathrm{CCH})$ is a major risk factor for vascular dementia (VaD). There are currently no broadly effective prevention or treatment strategies for $\mathrm{VaD}$, but recent studies have reported promising results following vascular bypass surgery and pharmacomodulation of the brain endocannabinoid system (ECS). In this study, early effects of encephalomyosynangiosis (EMS) bypass surgery and augmented endocannabinoid signaling on $\mathrm{CCH}$-induced cognitive dysfunction and neuronal damage were investigated.

Methods: An animal model of $\mathrm{VaD}$ was established by bilateral common carotid artery occlusion (BCCAO). Cannabinoid signaling was upregulated by treatment with the fatty acid amide hydrolase inhibitor URB597 (URB). Spatial learning and memory, cerebral blood flow (CBF), revascularization, brain-derived neurotrophic factor (BDNF)-tropomyosin receptor kinase B (TrkB) signaling, and apoptosis were compared among Sham, BCCAO, BCCAO + EMS, BCCAO + URB, and BCCAO + URB + EMS groups. Spatial learning and memory were evaluated using the Morris water maze (MWM). The CBF in cortex and hippocampus was evaluated by 3-dimensional arterial spin labeling. The neovascularization was visualized by CD34 immunofluorescence staining, and BDNF-TrkB signaling protein expression levels were assessed by Western blotting.

Results: Treatment with URB597 but not EMS alone reversed the spatial learning and memory deficits induced by BCCAO. Neovascularization was enhanced after EMS surgery but not by URB597. Alternatively, there were no significant differences in CBF among treatment groups. Expression levels of BDNF and TrkB were significantly reduced by $\mathrm{CCH}$ compared to Sham treatment, and downregulation of both proteins was reversed by URB597 treatment but not EMS. BCCAO enhanced neuronal apoptosis, which was also reversed by URB597.

Conclusions: Augmentation of endogenous cannabinoid signaling but not EMS protects against CCHinduced neurodegeneration and preserves spatial learning and memory, possibly by activating BDNF-TrkB signaling.

Keywords: BDNF; chronic cerebral hypoperfusion (CCH); cognitive dysfunction; encephalomyosynangiosis (EMS); endocannabinoid system (ECS)

Submitted Jun 02, 2020. Accepted for publication Nov 13, 2020.

doi: 10.21037/atm-20-4431

View this article at: http://dx.doi.org/10.21037/atm-20-4431 


\section{Introduction}

Chronic cerebral hypoperfusion $(\mathrm{CCH})$ is a hemodynamic abnormality common to multiple ischemic cerebrovascular diseases, including atherosclerosis, carotid stenosis/ occlusion, moyamoya disease (MMD), diabetic microangiopathy, and Alzheimer's disease (AD), all of which are characterized by cognitive and behavioral deficits with associated neurovascular damage collectively termed vascular dementia (VaD) $(1,2)$. These cognitive impairments result from neurodegeneration, apoptosis, and neuroinflammation secondary to $\mathrm{CCH}(3,4)$. Mild cognitive impairment, a relatively common age-related deficit (approximately $21.5-71.3$ per 1,000 person-years), is more likely to progress to $\mathrm{VaD}$ if associated with $\mathrm{CCH}(5,6)$. It was recently reported that 46.8 million people worldwide have dementia, and this number is expected to increase to 74.7 million by 2030 and 131.5 million by 2050 due to longevity gains (7). Thus, $\mathrm{CCH}$ contributes to the growing economic, social, and healthcare burdens associated with population aging, and there are no broadly effective treatments to ameliorate or slow down the progression of cognitive impairments induced by $\mathrm{CCH}$.

Extracranial-intracranial (EC-IC) vascular bypass procedures, including direct and indirect bypass surgeries, aim to restore partial perfusion in patients with cerebral ischemia (8). Encephalomyosynangiosis (EMS), an indirect bypass surgery, involves attaching the temporal muscle onto the surface of the ischemic cerebral cortex to establish collateral circulation $(9,10)$. It is a preferred procedure for children with MDD accompanied by episodes of ischemic symptoms (11). Some reports have demonstrated that EMS can improve neovascularization and decrease the incidence of ischemic or hemorrhagic cerebral vascular events $(12,13)$. However, the general efficacy of EMS is poor due to the delay in revascularization $(14,15)$. Further, the early and late effects of EMS surgery on cognitive impairments have not been systematically evaluated.

The endocannabinoid system (ECS) includes two cannabinoid ligands (anandamide and 2-arachidonoyl glycerol) and two cannabinoid receptors (CB1 and CB2), each regulated by various synthesizing and metabolizing enzymes such as fatty acid amide hydrolase (FAAH) (16). The ECS can modulate neural development, synaptic plasticity, and learning and memory $(17,18)$. Growing evidence indicates that modulation of ECS components has therapeutic potential against cognitive impairment related to $\mathrm{CCH}$ (19). For instance, FAAH inhibitors decreased infarct volume and enhanced synaptic transmission in a model of cerebral ischemia (20). Alternatively, however, other studies have suggested that the FAAH inhibitor 3'-carbamoylbiphenyl-3-yl cyclohexylcarbamate (URB597) actually causes cognitive decline and impairs long-term potentiation (LTP) $(21,22)$. Therefore, in this study, we investigated the effects and underlying mechanisms of EMS and URB597 treatment on CCH-induced cognitive dysfunction in a rat $\mathrm{VaD}$ model established by bilateral common carotid artery occlusion (BCCAO).

We present the following article in accordance with the ARRIVE reporting checklist (available at http://dx.doi. org/10.21037/atm-20-4431).

\section{Methods}

\section{Animals}

A total of 42 male Sprague Dawley rats $(300 \pm 10$ g, 1 month) were used in the present study. Animals were house in a climate-controlled facility $\left(24 \pm 1^{\circ} \mathrm{C}, 60 \%\right.$ humidity) under a 12-hour/12-hour light/dark cycle with free access to water and food. Experiments were performed under a project license (No. TJ20191012-A006) granted by institutional ethics board of Tongji University School of Medicine, in compliance with the National institutional guidelines for the care and use of animals.

\section{Surgical procedures and treatment groups}

The procedures for BCCAO and EMS surgery were conducted in accordance with previously published papers $(4,9)$. Rats were anesthetized with $0.15 \%$ pentobarbital sodium (40 mg/kg, i.p.), and placed in the supine position. An incision was made on the neck skin to expose bilateral internal carotid arteries, which were then carefully separated and ligated using 4-0 sutures, followed by EMS in the indicated groups (Figure 1). Briefly, the rats were turned to the prone position, a preauricular incision was made, and the right temporalis was separated from the skull. A microdiamond-tip cranial drill was used to open the temporal skull, and then a $1 \mathrm{~cm}^{2}$ craniectomy was performed. The dura was carefully separated from the brain surface to expose the cortex using microsurgical forceps under a dissection microscope. Temporal muscles were placed over the exposed cerebral region and sewn to the contralateral aponeurosis. The Sham-operated group was treated in the same manner without BCCAO and EMS. Two dead rats were excluded for later experiments after surgery. 

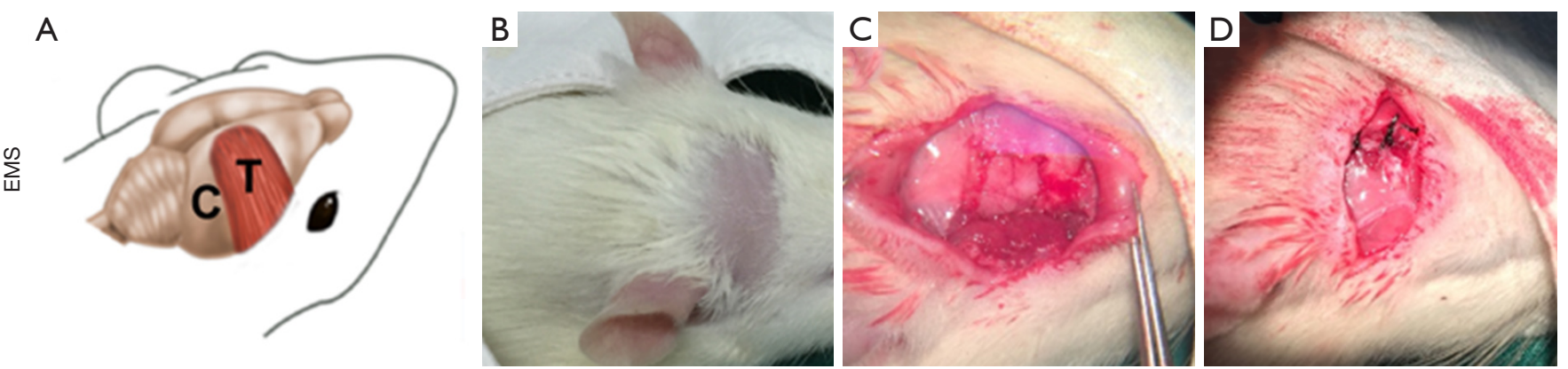

Figure 1 Encephalomyosynangiosis (EMS) surgery. (A) Schematic diagram of EMS. (B,C,D) Surgical procedure of EMS. C, cortex, T, temporalis.

After 1 week of feeding, 50 rats were randomly divided into 5 groups ( $\mathrm{n}=8$ in per group): [1] Sham, [2] BCCAO, [3] BCCAO with URB597 (BCCAO+URB), [4] BCCAO with EMS (BCCAO+EMS), and [5] BCCAO with cotreatment (BCCAO+URB+EMS). URB597 $\left(\mathrm{C}_{20} \mathrm{H}_{22} \mathrm{~N}_{2} \mathrm{O}_{3}\right)$ (Cayman Chemicals, Tallinn, Estonia) was administered via intraperitoneal (i.p.) injection at $0.3 \mathrm{mg} / \mathrm{kg} /$ day for 8 weeks after BCCAO surgery (23). Other groups were treated with an equal volume of $0.1 \%$ dimethyl sulfoxide vehicle.

\section{Morris water maze (MWM) task}

A MWM test pool, $1.8 \mathrm{~m}$ in diameter and $60 \mathrm{~cm}$ in height with a $20-\mathrm{cm}$ diameter escape platform, was filled with $24-26{ }^{\circ} \mathrm{C}$ opaque water. The escape platform was submerged $1 \mathrm{~cm}$ beneath the surface for place training. The pool was surrounded by a white curtain and divided into four quadrants marked with extra-visual cues. Rats were trained for 4 trials per day starting in one of the 4 quadrants for 4 consecutive days ( $\mathrm{n}=8$ rats per group). In each trial, rats were allowed to search for the hidden platform. If a rat did not find the platform in $60 \mathrm{~s}$, it was placed on the platform for $15 \mathrm{~s}$. The decrease in escape latency was taken as a measure of spatial learning. On day 5 , the platform was removed and rats were allowed to swim freely for $60 \mathrm{~s}$ in the probe trial. Time spent in the vicinity of the former platform location (target quadrant) and platform position crossings were used as measures of spatial memory. Data from the water maze test were recorded using a video camera and the Human Visual System Image Software (HVS Image, Hampton, UK).

\section{Three-dimensional arterial spin labeling}

Three-dimensional arterial spin labeling (3D-ASL), a noninvasive MRI perfusion assessment technique, was performed in accordance with previous reports (24). After anesthesia with $0.15 \%$ pentobarbital sodium $(40 \mathrm{mg} / \mathrm{kg}$, i.p.), rats ( $\mathrm{n}=8$ per group) were scanned on a $3.0 \mathrm{~T}$ Discovery MR750 (GE, Milwaukee, WI, USA) using an eight-channel wrist coil. The following $3 \mathrm{D}$-ASL imaging parameters were employed: field of view $=40 \mathrm{~mm} \times 20 \mathrm{~mm}$; matrix $=512$ (points) $\times 12$ (arms); slice thickness $=2 \mathrm{~mm}$; no gap between slices; 30 slices acquired in ascending order; flip angle $=90^{\circ}$; labeling duration $=1,650 \mathrm{~ms}$; post-labeling delay $=1,025 \mathrm{~ms}$; repetition time $=4,383 \mathrm{~ms}$; echo time $=11 \mathrm{~ms}$; number of excitations $=5$; bandwidth $=62.5 \mathrm{kHz}$; total scan duration $=9 \mathrm{~min} 47 \mathrm{~s}$. According to T2-weighted images, regions of interest (ROIs) were selected from the ipsilateral cortex and hippocampus. The same areas were selected to analyze cerebral blood flow (CBF). The CBF was calculated using the following equation $(24,25)$ :

$$
C B F=\frac{\lambda\left(1-e^{\frac{-t_{s a t}}{T_{1 g}}}\right)}{2 \alpha T_{1 b}\left(1-e^{\frac{-\tau}{T_{1 b}}}\right)} \frac{P W}{P D} e^{\frac{P L D}{T_{1 b}}}
$$

In the equation, $t_{\text {sat }}$ is the time of saturation before imaging $(2,000 \mathrm{~ms}), T_{1 \mathrm{~b}}$ is the $\mathrm{T}_{1}$ of blood $(1,600 \mathrm{~ms}), T_{1 \mathrm{~g}}$ is the $T_{1}$ of gray matter $(1,200 \mathrm{~ms}), \alpha$ is the labeling efficiency (0.95), $\lambda$ is the cortex blood partition coefficient (0.9), $\tau$ is the labeling duration $(1,500 \mathrm{~ms})$, and PLD is the delay time post-labeling (24). The CBF maps were automatically calculated by the GE medical scanner software (Functool 3D ASL, 4.5, Milwaukee, WI, USA).

\section{Immunofluorescent staining}

Brains ( $n=8$ per group) were fixed with $4 \%$ paraformaldehyde, embedded in paraffin, cut into $4-\mu \mathrm{m}$ 
thick slices, and deparaffinized. For antigen retrieval, slices were immersed in EDTA-Tris solution $(\mathrm{pH}$ 9.0) for $30 \mathrm{~min}$ at $96^{\circ} \mathrm{C}$. Tissue sections were then blocked with $10 \%$ normal goat serum in $0.01 \mathrm{M}$ phosphate-buffered saline at room temperature for $1 \mathrm{~h}$ and incubated with antiCD34 (1:200, ab81289, Abcam, MA, USA) overnight at $4{ }^{\circ} \mathrm{C}$, followed by incubation with a secondary antibody (Alexa Fluor 488-conjugated Ig, 1:300, Jackson Immunoresearch, USA) for $2 \mathrm{~h}$ at $37{ }^{\circ} \mathrm{C}$. After counterstaining with 4,6-diamidino-2-phenylindole (DAPI) for $8 \mathrm{~min}$ at $37^{\circ} \mathrm{C}$, the immunostaining was visualized by fluorescence microscopy at $200 \times$ magnification. For measurement of cerebral vessel numbers, three CD34-stained coronal sections from each rat were analyzed using Motic Image Advance 3.2 software (Motic, USA) (26). The numbers of CD34positive vessels were counted in five fields of view within the hippocampal CA1 area and the surgical area of cortex in each section. The average vessel number was quantified by two investigators blinded to treatment group.

\section{Western blot analysis}

Hippocampal and cortical tissues were extracted from rat brains ( $\mathrm{n}=8$ in per group) and homogenized in RIPA buffer (R0278, Sigma, St. Louis, MO, USA) at $4{ }^{\circ} \mathrm{C}$. Total protein was quantified using a bicinchoninic acid (BCA)based kit (BCA1, Sigma, St. Louis, MO, USA) and a spectrophotometer at $595 \mathrm{~nm}$ absorbance. Then, $30 \mu \mathrm{g}$ of total protein per sample was separated by $6-12 \%$ gradient sodium dodecyl sulfate polyacrylamide gel electrophoresis and transferred onto polyvinylidene difluoride membranes. The membranes were subsequently probed with primary antibodies against the following proteins overnight at $4{ }^{\circ} \mathrm{C}$ : brain derived neurotrophic factor (BDNF, 1:500, ab108319), glyceraldehyde-3-phosphate dehydrogenase (GAPDH, 1:5,000, ab8245) (both from Abcam, Cambridge, MA, USA), tropomyosin receptor kinase B (TrkB, 1:500, 4603S), poly-ADP ribose polymerase (PARP, 1:1,000, 9545S), and cysteinyl aspartate specific proteinase 3 (caspase-3, 1:500, 9664S) (all from Cell Signaling Technology, Beverly, MA, USA). Protein bands were visualized using an enhanced chemiluminescence detection kit (Millipore, Watford, UK), then normalized against the GAPDH level using ImageJ software.

\section{Statistical analysis}

All values are presented as mean \pm standard deviation (SD).
Multiple group means were compared by one-way or twoway ANOVA, followed by post hoc Tukey's tests for pairwise comparisons. Group and training day differences in MWM escape latencies were compared by repeatedmeasures ANOVA. All analyses were performed using SPSS 22.0 statistical software (IBM, USA). A P value $<0.05$ was considered statistically significant for all tests.

\section{Results}

\section{Upregulation of endogenous cannabinoid signaling but not EMS treatment rescued CCH-induced spatial memory deficits}

To assess possible deficits in spatial memory induced by $\mathrm{CCH}$ and amelioration by EMS and (or) upregulated cannabinoid signaling, performance in the MWM was compared among rats subjected to Sham treatment, BCCAO (CCH alone), BCCAO+EMS, BCCAO+URB, or $\mathrm{BCCAO}+\mathrm{EMS}+\mathrm{URB}$. Typical swim tracks during the probe trial are shown in Figure 2A. During the 4-day training period, rats in each group showed progressively shorter escape latencies, indicating spatial learning; however, escape latencies remained elevated in the BCCAO group compared to the Sham group $\left(\mathrm{F}_{4,160}=66.72, \mathrm{P}<0.001\right.$, Figure $2 \mathrm{~B}$ ), indicating that $\mathrm{CCH}$ impairs spatial learning in rats. By contrast, rats in both the BCCAO+URB and $\mathrm{BCCAO}+\mathrm{URB}+\mathrm{EMS}$ groups exhibited significantly shorter escape latencies than the BCCAO group (both $\mathrm{P}<0.05$; Figure $2 B$ ). In the probe trial, $\mathrm{BCCAO}+\mathrm{URB}$ and $\mathrm{BCCAO}+\mathrm{URB}+\mathrm{EMS}$ rats demonstrated greater numbers of platform crossings and spent more time in the target quadrant compared with the $\mathrm{BCCAO}$ group $(\mathrm{P}<0.05$, respectively) (Figure 2C,D). The $\mathrm{BCCAO}+\mathrm{EMS}$ group exhibited no significant differences in these probe trial measures compared to the $\mathrm{BCCAO}$ group, indicating no substantial restoration of spatial memory by EMS alone (Figure 2B,C,D). Indeed, both $\mathrm{BCCAO}$ and $\mathrm{BCCAO}+\mathrm{EMS}$ groups engaged in random aimless searches. Alternatively, $\mathrm{BCCAO}+\mathrm{URB}$ and $\mathrm{BCCAO+URB+EMS}$ groups showed significant preference for the target quadrant. The swimming speeds were similar among groups $\left(\mathrm{F}_{4,35}=1.343\right.$, $\mathrm{P}>0.05$, Figure $2 E$ ), indicating that motor deficits (and restoration of motor function) cannot explain these group differences in MWM performance. Notably, there were no statistical differences in number of platform crossings and time spent in the target quadrant between $\mathrm{BCCAO}+\mathrm{URB}$ and $\mathrm{BCCAO}+\mathrm{URB}+\mathrm{EMS}$ groups (Figure $2 C, D$ ). 

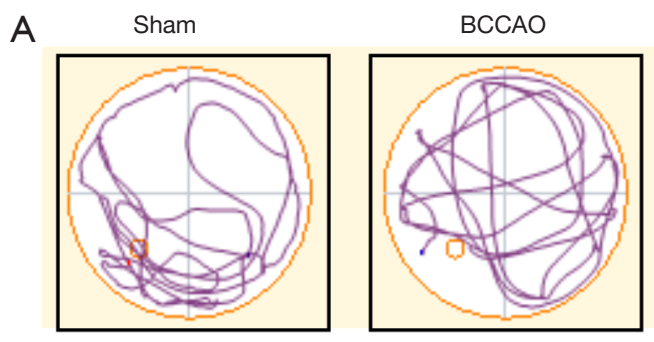

$B C C A O+$ EMS
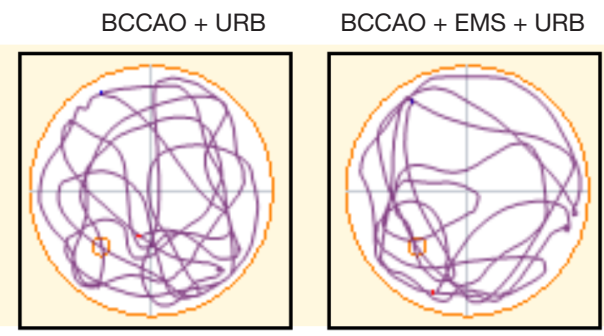

B

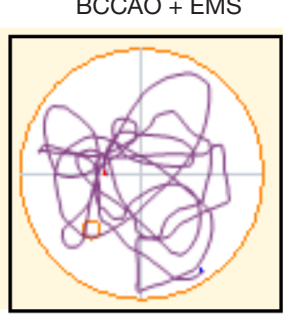

C
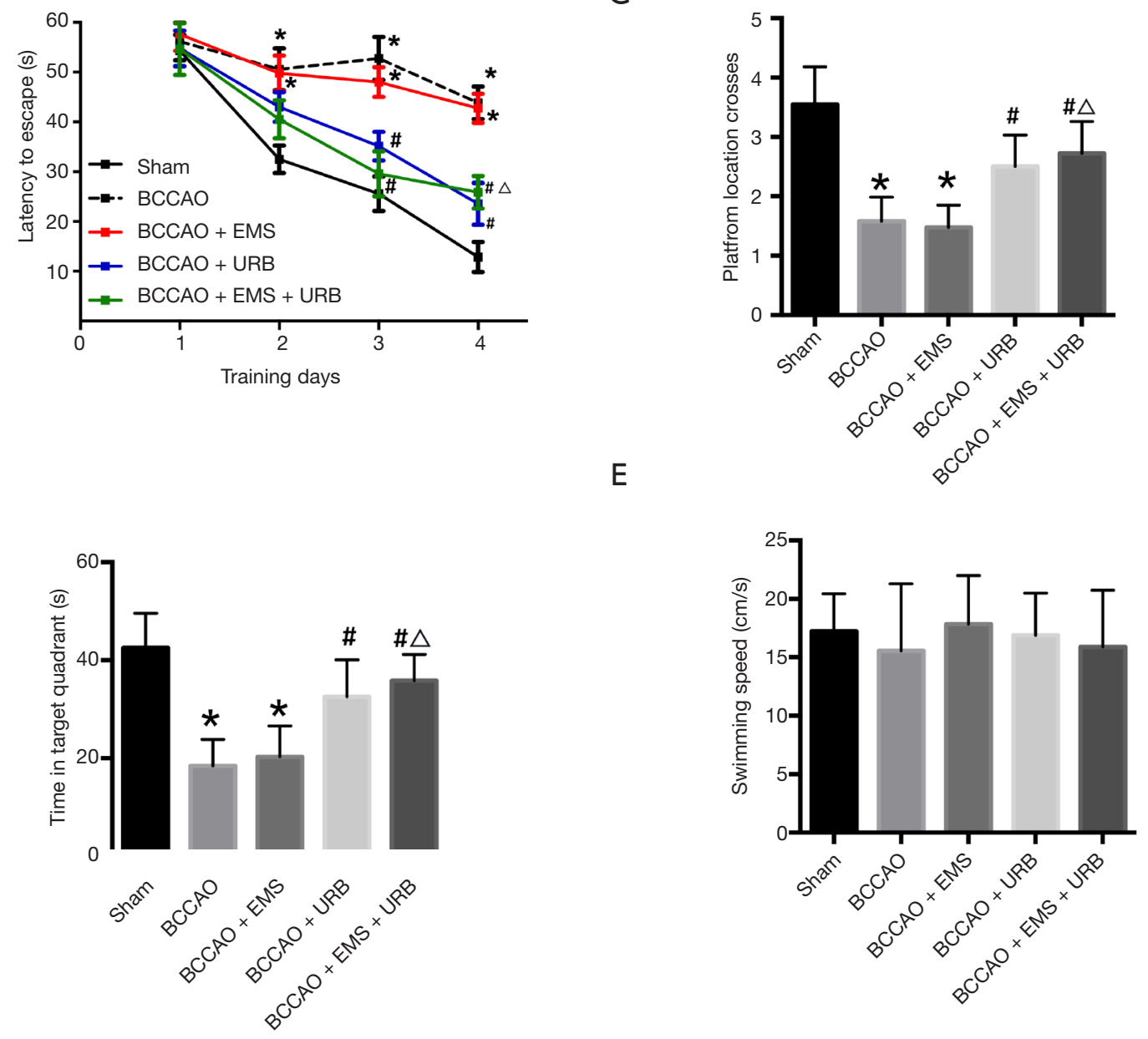

Figure 2 Effects of EMS and URB597 on chronic cerebral hypoperfusion (CCH)-induced spatial learning and memory deficits. (A) Representative swimming paths of rats in the probe trial. (B) Escape latencies during the training trials for the Sham, bilateral common carotid artery occlusion (BCCAO), BCCAO+EMS, BCAAO+URB, and BCAAO+URB+EMS groups. CCH markedly suppressed spatial learning (indicated by decreased escape latency), an effect reversed by URB597 (with or without EMS surgery) but not by EMS alone. (C,D) Numbers of platform location crossings and time spent in the target quadrant. These measures indicated significant impairment of spatial memory by $\mathrm{CCH}$ and reversal by URB597 but not EMS. (E) Swimming speed during the test. ${ }^{*} \mathrm{P}<0.05$ vs. Sham; ${ }^{*} \mathrm{P}<0.05$ vs. BCCAO; ${ }^{\triangle} \mathrm{P}<0.05$ vs. BCCAO+EMS. (B: $\left.\mathrm{F}_{4,160}=66.72, \mathrm{P}<0.001 ; \mathrm{C}: \mathrm{F}_{4,35}=9.324, \mathrm{P}<0.001 ; \mathrm{D}: \mathrm{F}_{4,35}=7.216, \mathrm{P}<0.01 ; \mathrm{E}: \mathrm{F}_{4,35}=1.343, \mathrm{P}>0.05\right)$. EMS, encephalomyosynangiosis. 
Collectively, these results suggest that EMS had no effects on spatial memory, while URB treatment can ameliorate $\mathrm{CCH}$-induced spatial learning and memory deficits.

\section{Changes in microvessel numbers (MVN)}

Fluorescence immunostaining for the endothelial cell marker CD34 revealed significant differences in neovascularization among groups $\left(\mathrm{F}_{4,35}=4.287, \mathrm{P}<0.05\right)$. Greater MVNs were found at the brain-temporal muscle interface of EMS group rats compared to the untreated cortex of BCCAO group rats (Figure $3 A, B$ ). Similarly, MVN was higher in $\mathrm{BCCAO}+\mathrm{URB}+\mathrm{EMS}$ rats than $\mathrm{URB}$ rats $(\mathrm{P}<0.05$; Figure $3 C)$. Alternatively, there were no obvious differences in hippocampal MVNs among groups. These results indicate that EMS increased MVN only in the ipsilateral operative region of the cortex.

\section{Effects of EMS and URB597 on CBF}

CBF was measured in the cortex and hippocampus using $3 \mathrm{D}-\mathrm{ASL}$ and mapped by pseudocolor. As shown in Figure $4 A, B$, the sagittal images were $2 \mathrm{~mm}$ caudal to bregma (red line). Quantitative analysis of CBF revealed the same pattern as shown in $3 \mathrm{D}$-ASL images. Predictably, BCCAO decreased CBF compared to the Sham group $(\mathrm{P}<0.01)$; surprisingly, however, there were no significant differences among treatment groups in either the cortex or hippocampus $\left(\mathrm{F}_{3,28}=1.229, \mathrm{P}>0.05 ; \mathrm{F}_{3,28}=1.308, \mathrm{P}>0.05\right.$, Figure $4 C, D)$. Thus, neither EMS nor URB597 improved $\mathrm{CBF}$ in cerebral areas with $\mathrm{CCH}$.

\section{Upregulated cannabinoid signaling but not EMS treatment rescued CCH-associated BDNF-TrkB signaling deficits}

We also studied effects on BDNF-TrkB signaling by measuring BDNF and TrkB expression by Western blotting (Figure 5A,B). Consistent with previous reports, BCCAO $(\mathrm{CCH})$ significantly reduced expression of BDNF and TrkB compared with the Sham group (both $\mathrm{P}<0.05$ ), and protein levels were not increased by EMS treatment. However, URB597 and EMS+URB597 reversed CCHinduced downregulation of BDNF and TrkB (BDNF, $\mathrm{F}_{4,35}=11.527, \mathrm{P}<0.001$, TrkB, $\mathrm{F}_{4,35}=4.413, \mathrm{P}<0.05$, Figure $5 C$; $\mathrm{BDNF}, \mathrm{F}_{4,35}=9.126, \mathrm{P}<0.001, \operatorname{TrkB}, \mathrm{F}_{4,35}=7.466, \mathrm{P}<0.01$, Figure 5D). Most importantly, there were no statistical differences between the BCCAO+URB and
BCCAO+URB+EMS groups, indicating that URB597 but not EMS upregulated BDNF-TrkB signaling in rats with $\mathrm{CCH}$.

\section{Neuroprotection by upregulation of endogenous cannabinoid signaling but not EMS treatment}

Preservation of spatial learning and memory by URB597 under $\mathrm{CCH}$ suggests protection of neurons from ischemic damage. To assess effects on neuronal viability, we compared neuronal apoptosis among groups by measuring PARP and the pro-apoptotic effector cleaved caspase-3 (Figure 6A,B). There were significant differences among groups (PARP, $\mathrm{F}_{4,35}=7.516, \mathrm{P}<0.01$, Caspase- $3, \mathrm{~F}_{4,35}=7.375$, $\mathrm{P}<0.01$, Figure 6C; PARP, $\mathrm{F}_{4,35}=4.338, \mathrm{P}<0.05$, Caspase -3 , $\mathrm{F}_{4,35}=8.015, \mathrm{P}<0.01$, Figure $\left.6 D\right)$. The expression levels of both proteins were significantly increased in the BCCAO and BCCAO+EMS groups compared with the Sham group. Upregulation of both proteins was reversed by URB597 (BCCAO+URB and BCCAO+URB+EMS groups) $(\mathrm{P}<0.05$ for both). Thus, URB597 but not EMS suppressed CCHinduced neuronal apoptosis.

\section{Discussion}

Extracranial-intracranial bypass surgery is an effective and safe revascularization procedure for chronic cerebral ischemia, and early surgical intervention can significantly reduce the incidence of ischemic events $(27,28)$. However, the effectiveness of EC-IC bypass surgery for long-term protection of cognitive function remains unclear. It was reported that cognitive impairments were significantly improved after direct bypass surgery in patients with occluded internal carotid arteries (29). However, the acute hemodynamic change in ischemic cerebral regions may contribute to new post-operative cognitive decline (30). Indirect bypass surgery may avoid rapid blood flow reversal by providing adaptable revascularization to reduce cerebral ischemia-associated neuronal damage (31). As in the EMS procedure, a temporal muscle graft is placed on the brain surface to serve as a source of collateral vessels promoting "on-demand" cranial vascular growth. In this study, neovascularization was demonstrated at the cortex-temporal muscle interface after EMS surgery, but not in URB597treated rats. However, angiogenesis occurs naturally during wound healing to support tissue growth and repair (32), which may account for the increased MVN after EMS surgery. 

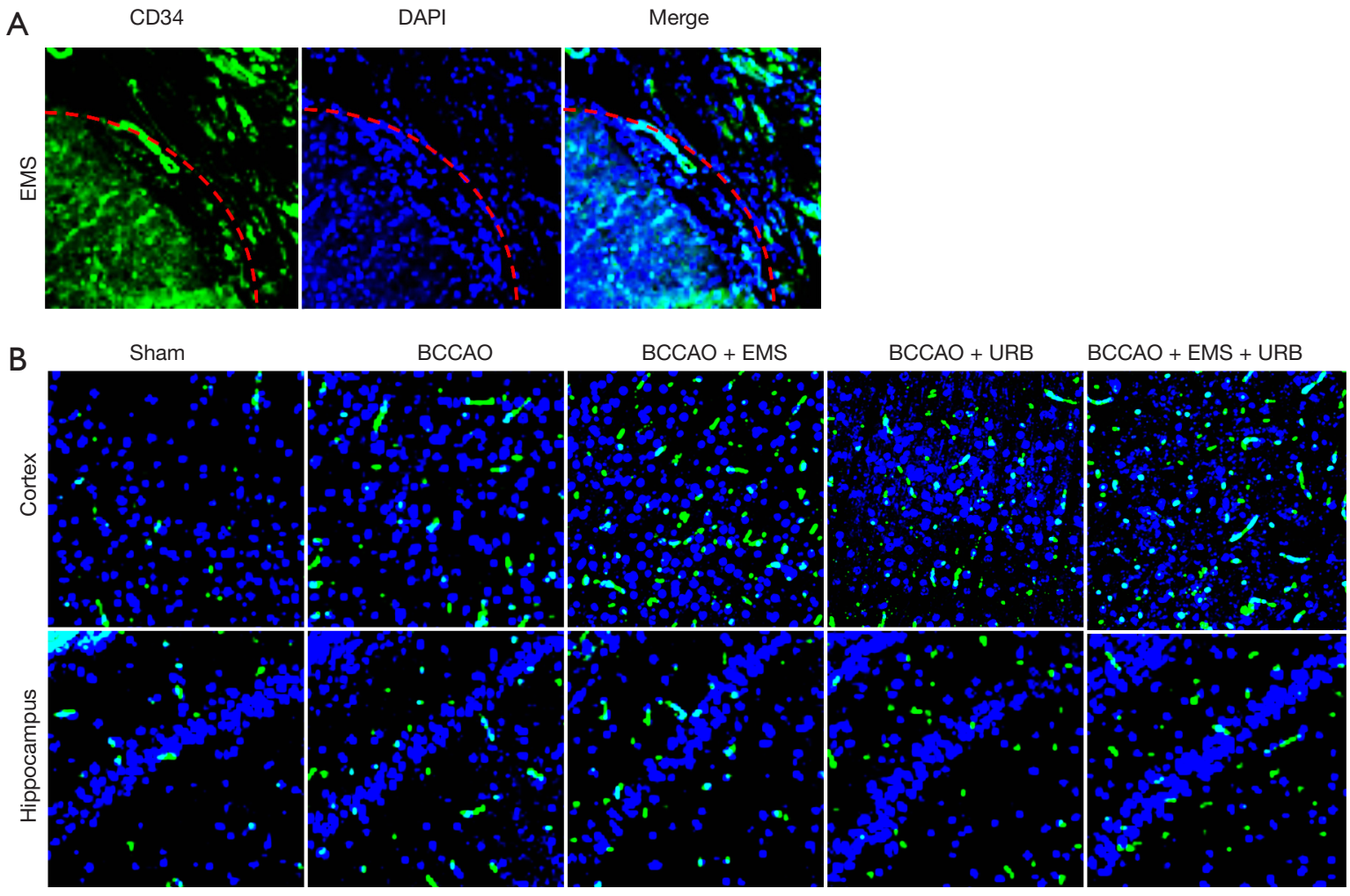

C
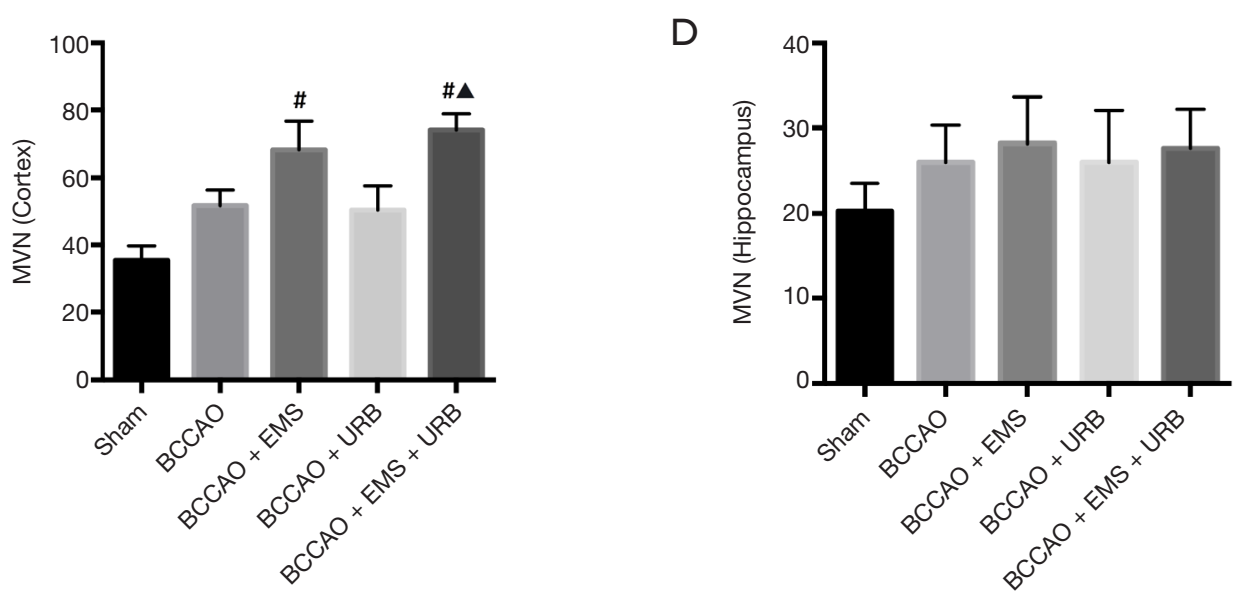

Figure 3 Effects of BCCAO, EMS, and URB597 on cortical and hippocampal neovascularization. (A) Immunopositivity to the endothelial cell marker CD34, indicative of neovascularization (green), was found at the cortical-temporal muscle interface following EMS surgery (red line) (magnification: 200×); (B) compared with the BCCAO group, the number of CD-34-positive microvessels (MVNs) was increased in the surgical area of cortex in EMS group rats (magnification: 200×). (C,D) Histogram showing MVN for each group. EMS but not URB957 enhanced MVN. ${ }^{*} \mathrm{P}<0.05$ vs. BCCAO; ${ }^{\mathbf{A}} \mathrm{P}<0.05$ vs. BCCAO+URB. $\left(\mathrm{C}: \mathrm{F}_{4,35}=4.287, \mathrm{P}<0.05 ; \mathrm{D}: \mathrm{F}_{3,28}=2.713, \mathrm{P}>0.05\right)$. EMS, encephalomyosynangiosis; BCCAO, bilateral common carotid artery occlusion. 
A

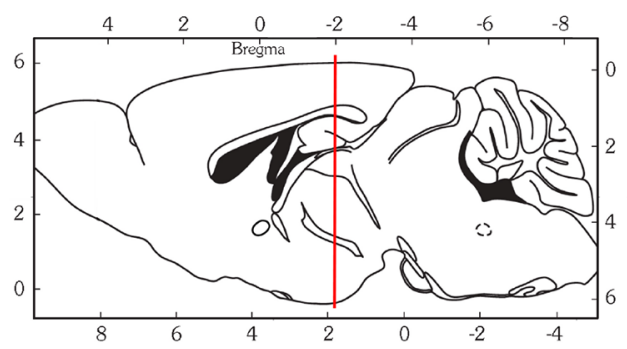

B

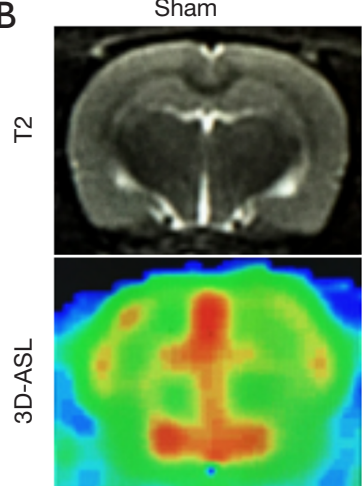

BCCAO

$B C C A O+E M S$

$\mathrm{BCCAO}+\mathrm{URB}$

$B C C A O+E M S+U R B$
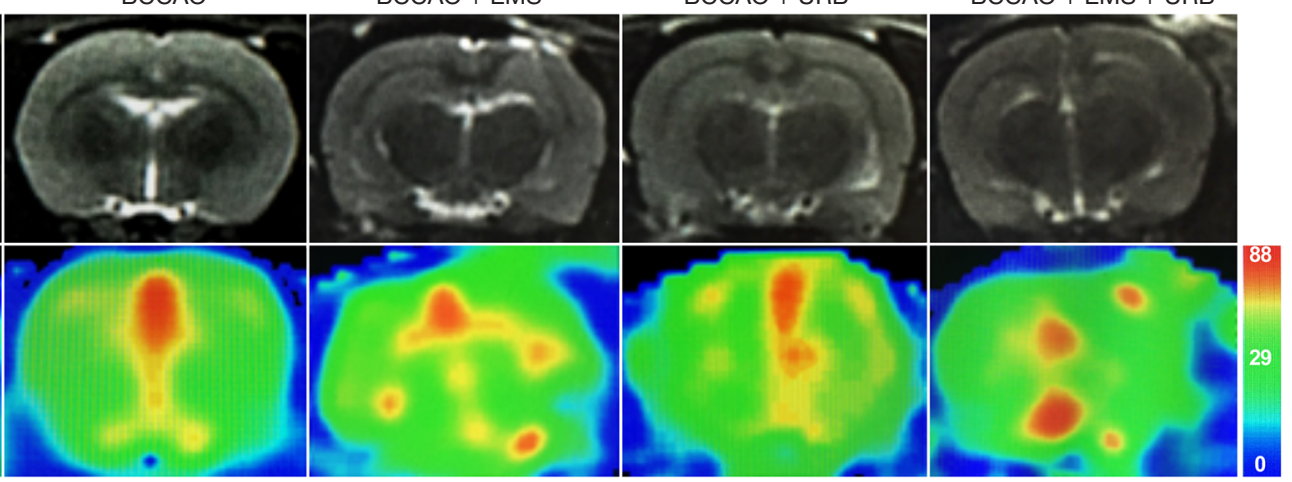

C
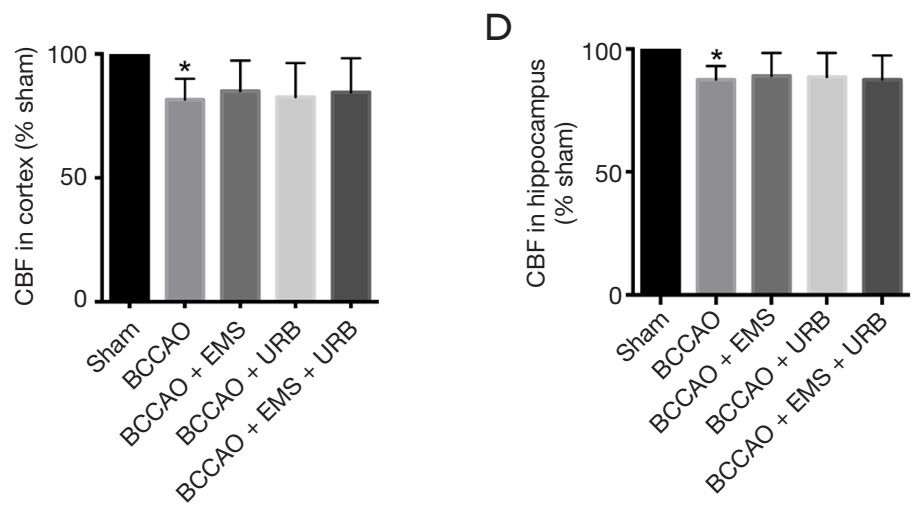

Figure 4 Effects of EMS and URB957 on the BCCAO-induced reduction in cerebral blood flow. (A) Sagittal images with anatomic coordinates. The red line is $2 \mathrm{~mm}$ caudal to bregma; (B) the level of T2 and 3D-ASL is selected (2 mm from bregma). Green represents the weakest and red the strongest CBF signal. (C,D) Quantitative analysis of CBF in the cortex and hippocampus. BCCAO significantly reduced CBF compared with the Sham group. Neither EMS nor URB597 reversed the effect of BCCAO. ${ }^{*} \mathrm{P}<0.05$ vs. Sham. (C: $\mathrm{F}_{3,28}=1.229, \mathrm{P}>0.05$; $\left.\mathrm{D}: \mathrm{F}_{3,28}=1.308, \mathrm{P}>0.05\right)$. EMS, encephalomyosynangiosis; BCCAO, bilateral common carotid artery occlusion; $\mathrm{CBF}$, cerebral blood flow.

Surgical revascularization aims at improving $\mathrm{CBF}$ in the ischemic area, but we found no significant differences across groups as measured by 3D-ASL. Thus, neither EMS nor URB597 increased CBF following CCH induction. Early research suggested good revascularization and increased CBF following EMS (8,33). Further, a more recent study reported that EMS provided efficient revascularization in children with MMD, suggesting therapeutic potential against $\mathrm{CCH}$ (11). The lack of EMS efficacy in the current study may be explained by the modest extent of revascularization prior to testing (e.g., MWM, CD34 staining), including the development of new vascular networks incorporating sinusoids, capillaries, venules, and arterioles, as these processes usually take more than 3 months $(15,34)$. Furthermore, long-term brain ischemia has differential effects on the length and pattern of microvessels. Compared to non-ischemic brain tissue, average vasculature length was significantly reduced in ischemia rat brain $(0.329 \pm 0.131$ vs. $\left.0.922 \pm 0.176 \mathrm{~m} / \mathrm{mm}^{3}\right)(35)$. New microvessels (i.e., capillaries and venules) can be categorized based on 
A

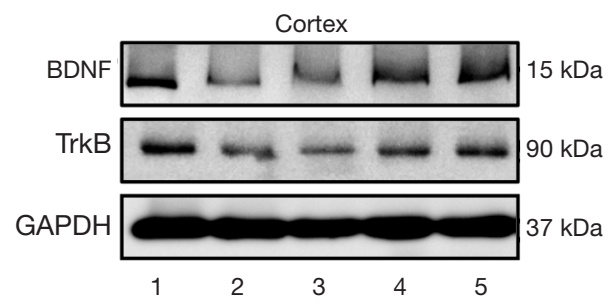

C

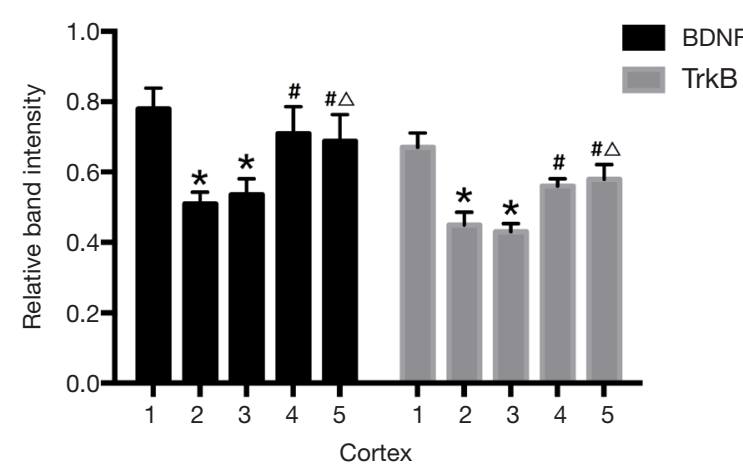

B

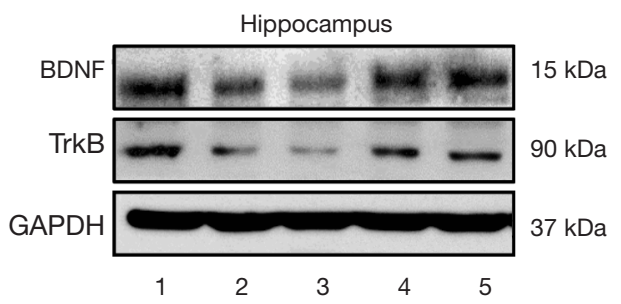

D

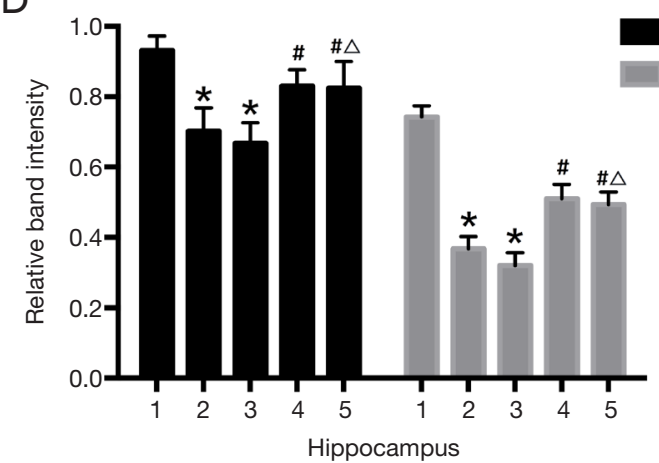

Figure 5 Effects of CCH, EMS, and URB597 on BDNF and TrkB expression levels. Lane numbers indicate protein samples from [1] Sham, [2] BCCAO, [3] BCCAO+EMS, [4] BCCAO+URB, and [5] BCCAO+EMS+URB groups. (A,B) Representative western blot of BDNF and TrkB; (C,D) quantitative analysis of protein expression. CCH reduced BDNF and TrkB expression compared with Sham surgery, effects reversed by URB597 (with or without EMS) but not by EMS alone. ${ }^{*} \mathrm{P}<0.05$ vs. Sham; ${ }^{~} \mathrm{P}<0.05$ vs. BCCAO; ${ }^{\triangle} \mathrm{P}<0.05$ vs. BCCAO+EMS. (C: BDNF, $\mathrm{F}_{4,35}=11.527, \mathrm{P}<0.001$, TrkB, $\mathrm{F}_{4,35}=4.413, \mathrm{P}<0.05$; D: BDNF, $\mathrm{F}_{4,35}=9.126, \mathrm{P}<0.001, \operatorname{TrkB}, \mathrm{F}_{4,35}=7.466, \mathrm{P}<0.01$ ). EMS, encephalomyosynangiosis; BCCAO, bilateral common carotid artery occlusion; $\mathrm{CCH}$, chronic cerebral hypoperfusion; BDNF, brainderived neurotrophic factor.

average diameter, such as capillaries $<10 \mu \mathrm{m}$ in diameter, smaller vessels from 10 to $20 \mu \mathrm{m}$ in diameter, and larger vessels $>20 \mu \mathrm{m}$ in diameter (36). Lugo-Hernandez et al. reported selective loss of capillaries, which accounted for $46.5 \%$ of the total microvessels, in ischemic tissue but no change was found in larger vessels (35). This finding may explain the relatively slow and autonomous CBF recovery regardless of treatment (37). In the current study, greater MVNs were found in the cortex and hippocampus of rats with $\mathrm{CCH}$, which is probably associated with $\mathrm{CCH}$-induced upregulation of VEGF mRNA and protein expression (38). Although this indirect bypass reduces the risk of recurrent hemorrhage in MMD, the ultimate functional outcome is less certain. Alternatively, treatment with URB597 (with or without EMS) improved spatial learning and memory compared to the BCCAO and BCCAO+EMS groups. Thus, despite a lack of improvement in CBF, promotion of cannabinoid signaling (but not EMS) reversed CCHinduced cognitive impairment.
The activity of the ECS is susceptible to modulation by pharmacological manipulation, such as by FAAH inhibitors (19). URB597 is a relatively selective inhibitor of FAAH that promotes ECS signaling by enhancing ligand and receptor levels (39). URB597 has demonstrated neuroprotective and anti-inflammatory properties and reverses LTP deficits in the hippocampus of aged rats without causing the undesirable side effects associated with $\mathrm{CB} 1$ and CB2 activation (40). Furthermore, URB597 enhanced recognition memory acquisition in $\mathrm{AD}$ model animals through CB2 activation and modulation of mammalian target of rapamycin (mTOR) signaling $(41,42)$. Recently, Biernacki et al. reported that inhibition of FAAH activity by URB597 increased anandamide and GPR55 receptor levels, thereby improving neuronal metabolism (43). In our previously study, URB597 reduced CCH-induced neural apoptosis by inhibiting CB1-mTOR-dependent autophagy and accumulation of reactive oxidative species, protective effects reversed by CB antagonist $(4,23,44)$. One 
A

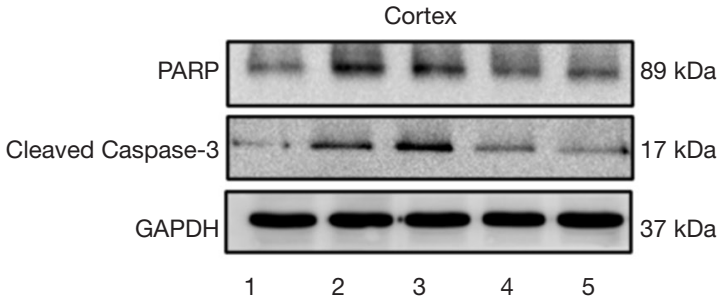

C

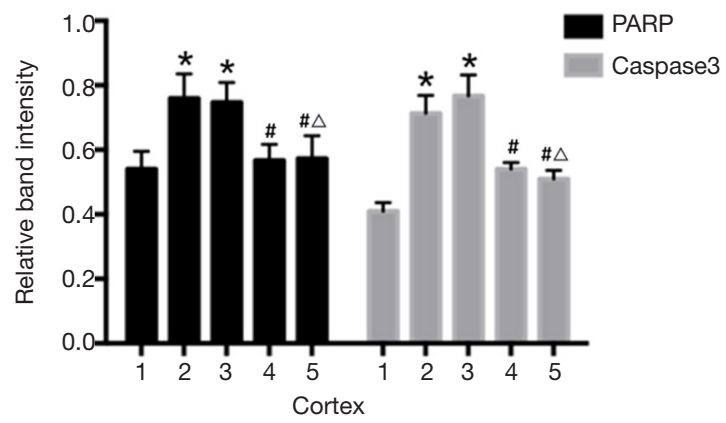

B

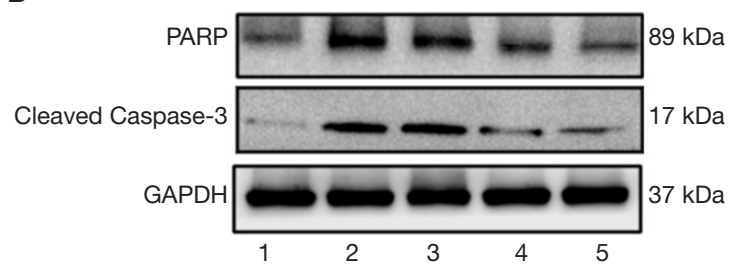

Hippocampus

D

Figure 6 Effects of EMS and URB597 on neuronal apoptosis. Apoptosis was estimated by expression of PARP and caspase-3. Numbers $1,2,3,4$, and 5 indicate the Sham, BCCAO, BCCAO+EMS, BCCAO+URB, and BCCAO+URB+EMS groups, respectively. (A,B) Representative western blot of PARP and caspase-3. (C,D) Quantitative analysis of protein expression. URB597 (with or without EMS) but not EMS alone reversed the reduction in expression induced by $\mathrm{CCH}$. ${ }^{*} \mathrm{P}<0.05$ vs. Sham; ${ }^{\#} \mathrm{P}<0.05$ vs. $\mathrm{BCCAO} ;{ }^{\circ} \mathrm{P}<0.05$ vs. $\mathrm{BCCAO}+\mathrm{EMS}$. (C: PARP, $\mathrm{F}_{4,35}=7.516, \mathrm{P}<0.01$, Caspase-3, $\mathrm{F}_{4,35}=7.375, \mathrm{P}<0.01$; D: PARP, $\mathrm{F}_{4,35}=4.338, \mathrm{P}<0.05$, caspase-3, $\left.\mathrm{F}_{4,35}=8.015, \mathrm{P}<0.01\right)$. EMS, encephalomyosynangiosis; BCCAO, bilateral common carotid artery occlusion; PARP, poly-ADP ribose polymerase.

clinical trial has demonstrated the efficacy and safety of a FAAH inhibitor in the treatment of cannabis withdrawal as evidenced by improvements in cognitive decline and withdrawal symptoms (45). A few studies have also suggested neuroprotection against $\mathrm{CCH}$ (46). In the current study, combination therapy with EMS and URB597 was not superior to URB597 treatment alone for suppressing apoptosis and improving spatial memory. In addition, numerous studies suggest that the ECS is involved in the control of arterial blood pressure and cardiac output, and participates in the regulation of regional vasoconstriction and vasodilation, as well as the blood supply to different organs, including the brain $(47,48)$. Vasodilation, a direct effect of ECS on cerebral vessels, is mediated, at least in part, by CB1 (47). URB597 can activate CB1, suggesting possible regulation of cerebrovascular resistance. CBF in ischemic cerebral region was enhanced only slightly by URB597, however, compared to the BCCAO group. We speculate that CBF regulation by URB597 is mild owing to the diverse functions of anandamine-CB1 signaling and the complexity of task-dependent cerebrovascular regulation. The effects of URB597 modulation may be highly dependent on dose and current physiological conditions.

The neurotrophin BDNF is a promoter of synaptic plasticity, neuronal development, and survival, and has been shown to reverse synapse loss and restore learning and memory in several disease models $(49,50)$. A deficiency of BDNF mRNA and protein has been observed in $\mathrm{AD}$ and $\mathrm{VaD}$, as well as in diabetes mellitus-induced dementia $(51,52)$. Hypoxia and ischemia also reduced BDNF expression in the mouse cerebral cortex (53). Similarly, the expression levels of BDNF and TrkB were significantly reduced by $\mathrm{CCH}$ compared with the Sham group, consistent with previous studies $(54,55)$. This reduction can result in neuronal apoptosis and inflammation (51). In contrast, enhancement of BDNF signaling by the cannabinoid receptor agonist WIN55, 212-2 ameliorated cognitive decline and synaptic plasticity (56). We previously showed that cannabinoid receptor agonists and URB597 improve cognitive impairment in $\mathrm{CCH}$ model rats via 
phosphatidylinositol 3-kinase/AKT signaling (44). The levels of BDNF and TrkB were dramatically increased after chronic treatment with URB597 and accompanied by a reversal in apoptosis and cognitive decline. The present findings reveal that URB597 can activate BDNF signaling and suppress apoptosis.

There are some limitations to this study, including the lack of individual Sham groups treated with EMS, URB, or URB+EMS. One clinical study reported that the FAAH inhibitor BIA 10-2474 may cause acute neurologic disorders (i.e., headache, cerebellar syndrome, and disturbance of consciousness) (57). Nonetheless, this did not mitigate the effect on learning and memory observed in the current study. Second, URB597 may have effects on anxiety or depression that benefit MWM performance (58). Therefore, future studies should include open-field, forced-swim, and learned helplessness tasks to assess such effects. In addition, microvasculature changes caused by $\mathrm{CCH}, \mathrm{URB}$, and EMS such as altered blood-brain barrier (BBB) function have not yet been studied. For this purpose, vascular tracer leakage assays and analyses of expression changes in BBB components, such as tight junction protein 1 and matrix metalloproteinases (59), should be conducted in further $\mathrm{CCH}$ studies.

In summary, we demonstrate for the first time that EMS has short-term protective effects against cognitive decline in a BCCAO model of $\mathrm{VaD}$, while URB597 acts a potential neuroprotective agent against $\mathrm{CCH}$-induced cognitive impairment, possibly by activating BDNF-TrkB signaling and attenuating ischemia-induced neuronal damage.

\section{Acknowledgments}

The authors thank Xiao-Liang Gong from Tongji University for assistance and support for 3D-ASL measurements.

Funding: The study was supported by the National Nature Science Foundation of China (Grant Nos. 81974207, 82001383), the Special Clinical Research Project of Shanghai Municipal Health Commission (20204Y0074), and Tong Ji Hospital Foundation (TJ1904).

\section{Footnote}

Reporting Checklist: The authors have completed the ARRIVE reporting checklist. Available at http://dx.doi. org/10.21037/atm-20-4431

Data Sharing Statement: Available at http://dx.doi. org/10.21037/atm-20-4431

Conflicts of Interest: All authors have completed the ICMJE uniform disclosure form (available at http://dx.doi. org/10.21037/atm-20-4431). The authors declare no conflicts of interest.

Ethical Statement: The authors are accountable for all aspects of the work in ensuring that questions related to the accuracy or integrity of any part of the work are appropriately investigated and resolved. Experiments were performed under a project license (No. TJ20191012-A006) granted by institutional ethics board of Tongji University School of Medicine, in compliance with the National institutional guidelines for the care and use of animals.

Open Access Statement: This is an Open Access article distributed in accordance with the Creative Commons Attribution-NonCommercial-NoDerivs 4.0 International License (CC BY-NC-ND 4.0), which permits the noncommercial replication and distribution of the article with the strict proviso that no changes or edits are made and the original work is properly cited (including links to both the formal publication through the relevant DOI and the license). See: https://creativecommons.org/licenses/by-nc-nd/4.0/.

\section{References}

1. Araki Y, Takagi Y, Ueda K, et al. Cognitive function of patients with adult moyamoya disease. J Stroke Cerebrovasc Dis 2014;23:1789-94.

2. Zhao Y, Gong CX. From chronic cerebral hypoperfusion to Alzheimer-like brain pathology and neurodegeneration. Cell Mol Neurobiol 2015;35:101-10.

3. Qiu L, Ng G, Tan EK, et al. Chronic cerebral hypoperfusion enhances Tau hyperphosphorylation and reduces autophagy in Alzheimer's disease mice. Sci Rep 2016;6:23964.

4. Wang D, Lin Q, Su S, et al. URB597 improves cognitive impairment induced by chronic cerebral hypoperfusion by inhibiting mTOR-dependent autophagy. Neuroscience 2017;344:293-304.

5. Eshkoor SA, Hamid TA, Mun CY, et al. Mild cognitive impairment and its management in older people. Clin Interv Aging 2015;10:687-93.

6. Langa KM, Levine DA. The diagnosis and management of mild cognitive impairment: a clinical review. JAMA 2014;312:2551-61. 
7. Wu Y'T, Beiser AS, Breteler MMB, et al. The changing prevalence and incidence of dementia over time - current evidence. Nat Rev Neurol 2017;13:327-39.

8. Czabanka M, Pena-Tapia P, Scharf J, et al. Characterization of direct and indirect cerebral revascularization for the treatment of European patients with moyamoya disease. Cerebrovasc Dis 2011;32:361-9.

9. Hecht N, Marushima A, Nieminen M, et al. Myoblast-mediated gene therapy improves functional collateralization in chronic cerebral hypoperfusion. Stroke 2015;46:203-11.

10. Hiramatsu M, Hishikawa T, Tokunaga K, et al. Combined gene therapy with vascular endothelial growth factor plus apelin in a chronic cerebral hypoperfusion model in rats. J Neurosurg 2017;127:679-86.

11. Imai H, Miyawaki S, Ono H, et al. The importance of encephalo-myo-synangiosis in surgical revascularization strategies for moyamoya disease in children and adults. World Neurosurg 2015;83:691-9.

12. Miyamoto S, Yoshimoto T, Hashimoto N, et al. Effects of extracranial-intracranial bypass for patients with hemorrhagic moyamoya disease: results of the Japan Adult Moyamoya Trial. Stroke 2014;45:1415-21.

13. Yanagihara W, Chida K, Kobayashi M, et al. Impact of cerebral blood flow changes due to arterial bypass surgery on cognitive function in adult patients with symptomatic ischemic moyamoya disease. J Neurosurg 2018;131:1716-24.

14. Powers WJ, Clarke WR, Grubb RL, Jr., et al. Extracranialintracranial bypass surgery for stroke prevention in hemodynamic cerebral ischemia: the Carotid Occlusion Surgery Study randomized trial. JAMA 2011;306:1983-92.

15. Thines L, Petyt G, Aguettaz P, et al. Surgical management of Moyamoya disease and syndrome: Current concepts and personal experience. Rev Neurol (Paris) 2015;171:31-44.

16. Iannotti FA, Di Marzo V, Petrosino S. Endocannabinoids and endocannabinoid-related mediators: Targets, metabolism and role in neurological disorders. Prog Lipid Res 2016;62:107-28.

17. Prospéro-García O, Ruiz Contreras AE, Ortega Gomez A, et al. Endocannabinoids as Therapeutic Targets. Arch Med Res 2019;50:518-26.

18. Patel S, Hill MN, Cheer JF, et al. The endocannabinoid system as a target for novel anxiolytic drugs. Neurosci Biobehav Rev 2017;76:56-66.

19. Fernández-Ruiz J, Moro MA, Martinez-Orgado J. Cannabinoids in Neurodegenerative Disorders and Stroke/Brain Trauma: From Preclinical Models to Clinical
Applications. Neurotherapeutics 2015;12:793-806.

20. Degn M, Lambertsen KL, Petersen G, et al. Changes in brain levels of $\mathrm{N}$-acylethanolamines and 2 -arachidonoylglycerol in focal cerebral ischemia in mice. J Neurochem 2007;103:1907-16.

21. Basavarajappa BS, Nagre NN, Xie S, et al. Elevation of endogenous anandamide impairs LTP, learning, and memory through CB1 receptor signaling in mice. Hippocampus 2014;24:808-18.

22. Kathuria S, Gaetani S, Fegley D, et al. Modulation of anxiety through blockade of anandamide hydrolysis. Nat Med 2003;9:76-81.

23. Wang DP, Liu KJ, Kasper G, et al. Inhibition of SENP3 by URB597 ameliorates neurovascular unit dysfunction in rats with chronic cerebral hypoperfusion. Biomed Pharmacother 2017;91:872-9.

24. Jing Z, Shi C, Zhu L, et al. Chronic cerebral hypoperfusion induces vascular plasticity and hemodynamics but also neuronal degeneration and cognitive impairment. J Cereb Blood Flow Metab 2015;35:1249-59.

25. Kim T, Kim SG. Quantification of cerebral arterial blood volume using arterial spin labeling with intravoxel incoherent motion-sensitive gradients. Magn Reson Med 2006;5 5:1047-57.

26. Yang J, Shi QD, Yang YB, et al. Vasoactive intestinal peptide administration after stroke in rats enhances neurogenesis and improves neurological function. Brain Res 2015;1625:189-97.

27. Rodríguez-Hernández A, Josephson SA, Langer D, et al. Bypass for the prevention of ischemic stroke. World Neurosurg 2011;76:S72-9.

28. Ando T, Shimada Y, Fujiwara S, et al. Revascularisation surgery improves cognition in adult patients with moyamoya disease. J Neurol Neurosurg Psychiatry 2020;91:332-4.

29. Fiedler J, Priban V, Skoda O, et al. Cognitive outcome after EC-IC bypass surgery in hemodynamic cerebral ischemia. Acta Neurochir (Wien) 2011;153:1303-11; discussion 1311-2.

30. Inoue T, Ohwaki K, Tamura A, et al. Postoperative transient neurological symptoms and chronic subdural hematoma after extracranial-intracranial bypass for internal carotid/middle cerebral atherosclerotic steno-occlusive diseases: negative effect on cognitive performance. Acta Neurochir (Wien) 2016;158:207-16.

31. Komotar RJ, Starke RM, Otten ML, et al. The role of indirect extracranial-intracranial bypass in the treatment of symptomatic intracranial atheroocclusive disease. J 
Neurosurg 2009;110:896-904.

32. Silvestre JS, Smadja DM, Levy BI. Postischemic revascularization: from cellular and molecular mechanisms to clinical applications. Physiol Rev 2013;93:1743-802.

33. Hecht N, Pena-Tapia P, Vinci M, et al. Myoblast-mediated gene therapy via encephalomyosynangiosis--a novel strategy for local delivery of gene products to the brain surface. J Neurosci Methods 2011;201:61-6.

34. Nakamura M, Imai H, Konno K, et al. Experimental investigation of encephalomyosynangiosis using gyrencephalic brain of the miniature pig: histopathological evaluation of dynamic reconstruction of vessels for functional anastomosis. Laboratory investigation. J Neurosurg Pediatr 2009;3:488-95.

35. Lugo-Hernandez E, Squire A, Hagemann N, et al. 3D visualization and quantification of microvessels in the whole ischemic mouse brain using solvent-based clearing and light sheet microscopy. J Cereb Blood Flow Metab 2017;37:3355-67.

36. Paul D, Cowan AE, Ge S, et al. Novel 3D analysis of Claudin-5 reveals significant endothelial heterogeneity among CNS microvessels. Microvasc Res 2013;86:1-10.

37. Zou W, Song Y, Li Y, et al. The Role of Autophagy in the Correlation Between Neuron Damage and Cognitive Impairment in Rat Chronic Cerebral Hypoperfusion. Mol Neurobiol 2018;55:776-91.

38. Hai J, Li ST, Lin Q, et al. Vascular endothelial growth factor expression and angiogenesis induced by chronic cerebral hypoperfusion in rat brain. Neurosurgery 2003;53:963-70; discussion 70-2.

39. Bedse G, Romano A, Lavecchia AM, et al. The role of endocannabinoid signaling in the molecular mechanisms of neurodegeneration in Alzheimer's disease. J Alzheimers Dis 2015;43:1115-36.

40. Murphy N, Cowley TR, Blau CW, et al. The fatty acid amide hydrolase inhibitor URB597 exerts antiinflammatory effects in hippocampus of aged rats and restores an age-related deficit in long-term potentiation. J Neuroinflammation 2012;9:79.

41. Ratano P, Petrella C, Forti F, et al. Pharmacological inhibition of 2-arachidonoilglycerol hydrolysis enhances memory consolidation in rats through CB2 receptor activation and $\mathrm{mTOR}$ signaling modulation. Neuropharmacology 2018;138:210-8.

42. Hasanein P, Teimuri Far M. Effects of URB597 as an inhibitor of fatty acid amide hydrolase on WIN55, 212-2-induced learning and memory deficits in rats. Pharmacol Biochem Behav 2015;131:130-5.
43. Biernacki M, Baranowska-Kuczko M, Niklinska GN, et al. The FAAH Inhibitor URB597 Modulates Lipid Mediators in the Brain of Rats with Spontaneous Hypertension. Biomolecules 2020;10:1022.

44. Su SH, Wang YQ, Wu YF, et al. Cannabinoid receptor agonist WIN5 5,212-2 and fatty acid amide hydrolase inhibitor URB597 may protect against cognitive impairment in rats of chronic cerebral hypoperfusion via PI3K/AKT signaling. Behav Brain Res 2016;313:334-44.

45. D'Souza DC, Cortes-Briones J, Creatura G, et al. Efficacy and safety of a fatty acid amide hydrolase inhibitor (PF04457845) in the treatment of cannabis withdrawal and dependence in men: a double-blind, placebo-controlled, parallel group, phase 2a single-site randomised controlled trial. Lancet Psychiatry 2019;6:35-45.

46. Su SH, Wu YF, Lin Q, et al. Cannabinoid receptor agonist WIN55,212-2 and fatty acid amide hydrolase inhibitor URB597 suppress chronic cerebral hypoperfusion-induced neuronal apoptosis by inhibiting c-Jun $\mathrm{N}$-terminal kinase signaling. Neuroscience 2015;301:563-75.

47. Benyó Z, Ruisanchez E, Leszl-Ishiguro M, et al. Endocannabinoids in cerebrovascular regulation. Am J Physiol Heart Circ Physiol 2016;310:H785-801.

48. Stanley C, O'Sullivan SE. Vascular targets for cannabinoids: animal and human studies. Br J Pharmacol 2014;171:1361-78.

49. Park H, Poo MM. Neurotrophin regulation of neural circuit development and function. Nat Rev Neurosci 2013;14:7-23.

50. Wan Q, Ma X, Zhang ZJ, et al. Ginsenoside Reduces Cognitive Impairment During Chronic Cerebral Hypoperfusion Through Brain-Derived Neurotrophic Factor Regulated by Epigenetic Modulation. Mol Neurobiol 2017;54:2889-900.

51. Shang J, Yamashita T, Zhai Y, et al. Strong Impact of Chronic Cerebral Hypoperfusion on Neurovascular Unit, Cerebrovascular Remodeling, and Neurovascular Trophic Coupling in Alzheimer's Disease Model Mouse. J Alzheimers Dis 2016;52:113-26.

52. Kwon KJ, Lee EJ, Kim MK, et al. Diabetes augments cognitive dysfunction in chronic cerebral hypoperfusion by increasing neuronal cell death: implication of cilostazol for diabetes mellitus-induced dementia. Neurobiol Dis 2015;73:12-23.

53. Tian X, Hua F, Sandhu HK, et al. Effect of delta-opioid receptor activation on $\mathrm{BDNF}-\mathrm{TrkB}$ vs. TNF-alpha in the mouse cortex exposed to prolonged hypoxia. Int J Mol Sci 2013;14:15959-76. 
54. Sakr HF, Khalil KI, Hussein AM, et al. Effect of dehydroepiandrosterone (DHEA) on memory and brain derived neurotrophic factor (BDNF) in a rat model of vascular dementia. J Physiol Pharmacol 2014;65:41-53.

55. Wu X, Sun J, Zhang X, et al. Epigenetic signature of chronic cerebral hypoperfusion and beneficial effects of S-adenosylmethionine in rats. Mol Neurobiol 2014;50:839-51.

56. Talani G, Licheri V, Biggio F, et al. Enhanced Glutamatergic Synaptic Plasticity in the Hippocampal CA1 Field of Food-Restricted Rats: Involvement of CB1 Receptors. Neuropsychopharmacology 2016;41:1308-18.

Cite this article as: Wang DP, Lin Q, Kang K, Wu YF, Su SH, Hai J. Preservation of spatial memory and neuroprotection by the fatty acid amide hydrolase inhibitor URB597 in a rat model of vascular dementia. Ann Transl Med 2021;9(3):228. doi: 10.21037/atm-20-4431
57. Kerbrat A, Ferre JC, Fillatre P, et al. Acute Neurologic Disorder from an Inhibitor of Fatty Acid Amide Hydrolase. N Engl J Med 2016;375:1717-25.

58. Danandeh A, Vozella V, Lim J, et al. Effects of fatty acid amide hydrolase inhibitor URB597 in a rat model of trauma-induced long-term anxiety. Psychopharmacology (Berl) 2018;235:3211-21.

59. Qu C, Xu L, Shen J, et al. Protection of bloodbrain barrier as a potential mechanism for enriched environments to improve cognitive impairment caused by chronic cerebral hypoperfusion. Behav Brain Res 2020;379:112385. 